salicylic acid on echinoderm division (Smith, 1925) are examples where the ion is entirely or almost entirely inactive.

A number of drugs, particularly the alkaloids and local anæsthetics, have $p \mathrm{~K}$ volues between 6 and 8 . This means that at physiological values of hydrogen ion concentration (around $p H$ 7), both ions and neutral molecules are present in a ratio lying between 1 to 1 and 1 to 9 . Such drugs can pass through membranes which would exclude ions and yet can regenerate some ions (by mass action) on the far side of the membrane.

From these and other examples, it is clearly important to know the ionization constants of all biologically active substances under investigation. Such knowledge should then be used to decide whether ion or molecule is the more active. This is done in two complementary ways: (i) by varying the $p \mathrm{~K}$ of the drug through appropriate substitution, keeping the $p \mathbf{H}$ of the biological medium constant; (ii) by keeping the composition of the drug constant and varying the $p H$. Such information can materially shorten the time taken to find the most active substance in a given series, because it brings one of the commoner variables completely under control.

Drs. H. J. Barber, F. Bergel, T. McLachlan, B. A. Pethica, M. A. Phillips, D. Ridge, J. H. Schulman, R. Slack and H. I. Stonehill took part in the discussion.

AdRIEN ALBerT

\section{OPERATIONAL ANALYSIS AND THE NATURE OF SOME PHYSICAL CONCEPTS}

He Philosophy of Science Group of the British Society for the History of Science, under the chairmanship of Prof. J. H. Woodger, held a meeting on May 5 at University College, London. A general discussion took place on questions arising out of Prof. P. W. Bridgman's three lectures on "The Nature of Some of our Physical Concepts" delivered at University College during April 24-28. (These lectures are to be published in the British Journal for the Philosophy of Science.) The meeting was attended by about forty members of the Group and visitors.

Prof. Bridgman opened the discussion with a brief summary of his lectures. He thought that their most important feature was the detailed analysis of how the operational technique can be applied to specific physical concepts and problems. In the first lecture, he had discussed general concepts such as field, action at a distance, and empty space; in the second, thermodynamical phenomena, in particular the laws relating to energy and entropy; and in the third, some situations presented by thermo-electric phenomena. Prof. Bridgman believed that the particular novelty of his analysis in these lectures was the self-conscious separation of physical operations into those of an instrumental character and those of a 'paper-and-pencil'--that is, mentalcharacter. He had been aware of this distinction for a long time, but only recently had he seen how it applied to what physicists actually do. With regard to this, there are two points of view, as follows.

Instrumental operations correspond most nearly to what used to be called 'physical reality'. This aspect of physics can be illustrated by an analysis of the field concept. Instrumentally, the existence of a field at a certain place means that, if you go there with the appropriate type of instrument, it will record a certain 'reading'. Similarly, in applying the first law of thermodynamics, one must specify the elements of space, and in particular their boundaries, on which one operates, so that the fluxes over these boundaries correspond to the actual instrumental readings.

The other aspect relates to the way in which our verbal demands interlock with our instrumental operations. For example, we accept the typical conservation laws of physics as verbal guides with rational implications, but we also find that there exist instrumental operations corresponding to our verbal demands.

It is Prof. Bridgman's belief that, in general, no sharp dividing line can be drawn in physics between the purely instrumental and the purely mental. $\mathrm{He}$ regards the whole operational approach as part of a larger programme, according to which we regard the world about us in terms of 'activity' rather than picturing it statically. From this point of view, it is preferable to describe our theories in terms of what we 'do' rather than in terms of 'things'.

Prof. H. Dingle said that, although he is in general agreement with the operational point of view, he attaches less importance than Prof. Bridgman to the distinction between instrumental and paper-andpencil operations. He prefers to emphasize instead the distinction between those concepts which are associated with measurements, usually denoted by symbols in our equations, and those, such as action at a distance, empty space, ete, which we employ 'pictorially'. He thought that this differentiation is important because we should confine our operational analysis to concepts of the former type, as he has already explained in some detail in his presidential address to the Group in March 1949 (Brit. $J$. Phil. Sci., 1, $5 ; 1950$ ). Prof. Dingle considered that one of the most important applications of the operational principle concerns the separation of the actual experimental results from the theoretical concepts used in visualizing them. As an example, he directed attention to a well-known method of determining the wave-length of monochromatic light by means of a diffraetion grating. The fundamental equation can be written as $d \sin \theta=m \lambda$, as explained in standard text-books. Operationally, the numbers $d, \theta$ and $m$ (spectrum number) can be directly determined; but the fourth number $\lambda$, the wave-length, is never measured directly, that is, from crest to crest. Hence, the operational content of this equation is confined to the invariance of the quantity $\bar{d} \sin \theta / m$ for different values of $d, \theta$ and $m$. If we wish, we can denote this invariant by the symbol $\lambda$. So far, no picture of this invariance need be formed. If, however, we appeal now to theory and regard light as consisting of trains of waves, we can give a theoretical interpretation to $\lambda$ as a length; whereas, if we regard light as consisting of corpuscles, we can interpret $\lambda$ as a constant of the dimensions of action divided by momentum. We are at liberty to use any picture we find satisfactory, provided that $\lambda$ has the correct physical dimensions. The function of such pictures is to stimulate and guide research, to facilitate the mental grasp of the situation, and ultimately to disappear in favour of better ones. These functions are not assisted but rather hindered by the demand that the details of the pictures must satisfy the operational criterion. 
Turning to a point of detail, Prof. Dingle said that he had not been convinced by Prof. Bridgman's argument that energy can be located, as it appeared to be at variance with Prof. Bridgman's own demonstration that light cannot be located in transit, but only at points of emission and absorption.

Prof. Bridgman, said that he regarded $\lambda$ as having a larger instrumental component than Prof. Dingle had indicated. He thought that Prof. Dingle had considered this particular question from too narrow a point of viow and had omitted the fact that a definite $\lambda$ is to be correlated with a definite source. The same source can be used for many other experiments, for example, diffraction, Newton's rings, etc., yielding the same $\lambda$. This invariant would then be regarded as a fundamental characteristic of the source having not only paper-and-pencil, but also instrumental, significance. We can both usefully calculate with $\lambda$ as a constant in theoretical work and also connect $\lambda$ with the instrumental specification of the source. Consequently, he thought that in a wider setting Prof. Dingle's sharp distinction between the instrumental and the mental components would become blurred.

With regard to the specific question about energy and light, Prof. Bridgman remarked that he was glad to have the opportunity of disowning his former view, expressed more than twenty years ago in his book "The Logic of Modern Physics", that energy is not localized. He thought that the localization of energy in a beam of light becomes possible when we can determine the microscopic $E$ - and $H$-vectors in the beam. as they vary during a period, and can thus determine the fine-scale Poynting vector.

The question came from Mr. C. C. L. Gregory, who inquired about the application of operational analysis to the interior of stars. In view of the practical difficulty of determining temperatures, etc., in such regions, he asked if we ought to regard the subject of stellar structure, at least in so far as it relates to internal constitution, as wholly of a paper-and-pencil character. More generally, he wondered whether physioists do not often assume implicitly that operations can be done instrumentally which, in fact, cannot be so done. He also wondered whether the whole concept of change does not entail difficulties as regards the application of our paper-and-pencil concepts. Certainly, we seem to base physical theories as far as possible on conservation laws which correspond to essentially mental demands.

Dr. G. J. Whitrow agreed that we often tend to regard certain impracticable instrumental processes as possible 'in principle' when, strictly speaking, they are not even that. In Prof. Bridgman's discussion concerning our inability to distinguish instrumentally between the concepts of field and action at a distance (with the conclusions of which he fully agreed), he thought that just such an example had been introduced. It has been suggested that gravitational force, whether due to a field or to action at a distance, can in principle be detected instrumentally in a region midway between the earth and the sun. He thought that there is a difficulty here, over and above that arising from the inaccessibility of such a region. In time such a region may cease to be inaccessible, but, unless special precautions can be taken, the instrument concerned will be influenced in the same way as the actual test-mass to be introduced in this region and so will give no 'reading'. The necessary precautions for counteracting the gravitational effect on the instrument can, of course, be taken on the (almost) rigid surface of the earth, but he thought that in inter-planetary space the situation is very different. In general, he considered that the farther one goes from terrestrial laboratories, etc., the more predominant becomes the paper-and-pencil technique in physies, while the instrumental element tends to be restricted to kinematical rather than dynamical phenomena. In reply, Prof. Bridgman said that he had, of course, intended that the instrument used should be 'anchored' to the sun, and he thought that no difficulty arose in principle. Dr. Whitrow considered that this was so impracticable that he found it difficult to imagine that any instmument could be effectively shielded against the gravitational influence of the sun. In subsequent discussion, Prof. K. R. Popper suggested that a proper platform for the gravitation-indicating instrument could in principle be provided by a rocket so controlled as to be moving with uniform velocity relative to the sun.

Dr. W. Ehrenberg asked whether the view that energy is localized would imply that the field concept is right and action at a distance wrong. Prof. Bridg. man thought that in any given case a problem such as this can only be answered by carrying out an analysis which will show in detail what the localization is. With regard to gravitation, he thought that the energy can consistently be located in the massive bodies, just as an alternative mathematical method of localizing electrostatic energy is in the charges. If there were not a magnetic field surrounding an electrostatic charge in motion, there would be no Poynting vector. The magnetic field about a moving charge is so small that it is difficult to detect instrumentally when charges are displaced, and until it was discovered energy had to be localized in the charges. But with the discovery of the mere existence of this very small effect, the picture changes qualitatively, and we now visualize the energy as diffused throughout space. A perfectly consistent picture of the localization of gravitational energy in the masses can be built up.

In reply to a question by Dr. E. H. Hutten, concerning entropy and irreversible thermodynamic processes, Prof. Bridgman said that bodies exist for which every departure from a given configuration is irreversible. He has posed the problem of inventing a generalized entropy function which would be universally applicable and would continually increase for the universe. Dr. Hutten agreed that in Nature there are no truly reversible processes, and that, strictly spealking, the classical entropy concept applies only to abstract processes.

Concerning the fundamental question of the meaning of operational analysis, Dr. Hutten thought that it is important to distinguish between thoughtexperiments which are essentially of a paper-andpencil character and others, for example, those relating to the far side of the moon, which are only so because of our present technical limitations. He suggested that, on the lines of the traditional 'synthetic-analytic' division of thought, we should describe the division now contemplated as 'semanticsyntactic'. Prof. Bridgman, however, did not favour a rigid division, and gave it as his opinion that one must work in a laboratory in order to understand fully what operational analysis is.

It was suggested by Dr. M. D. H. Strauss that there are two main uses of operational analysis, namely, for clarifying a given theory, and also for helping to delimit the field of application of a theory. Often a restricted domain of phenomena can be 
described theoretically in a number of different ways which are operationally equivalent, but when a wider domain is considered some of the competing theories will tend to be discarded. Maxwell's equations, for example, are not applicable to the elementary particles. Prof. Bridgman said that his own remarks concerning these equations applied only to ponderable matter. He thought that, when attempting to construct general theories in physics, it is a mistake to be dominated by fixed intuitions. In his opinion, Einstein made a mistake of this kind in constructing his generalized theory of gravitation, for his point of view was dictated by his intuition concerning the universal application of the field concept.

Mr. L. L. Whyte thought, however, that one of the main functions of operational analysis is its use in clarifying basic principles in physics with the object of extending their field of application. Einstein and Heisenberg, for example, each made a kind of operational analysis directed to differentiating between the measurable and the inherited mental components in certain branches of physics. Their work was essentially positive and creative, for they discovered elements in our thought which were inessential. Our thought became more powerful when these elements were discarded. On a point of detail, Mr. Whyte said that he believed that a thoroughgoing operational analysis should be made of the concept of time in quantum physics. A direct appeal to time is not usually made in determining, say, the velocity of an electron, although in recent work with electron tubes direct time effects are observed. Prof. Bridgman agreed that this whole question ought to be analysed much more fully than it had been hitherto.

In reply to a further question by $\mathrm{Mr}$. Whyte, Prof. Bridgman said that he had worked on high pressure physics before he began to study operational analysis. This study arose out of a distinct response to his own personal experience; it grew out of his essay on dimensional analysis. It was greatly stimulated by his being suddenly called upon to give a course of lectures on electrodynamics, and so being confronted with the need to think matters out anew for himself.

$$
\text { G: J. WHITRow }
$$

\section{ANTIBIOTICS IN THE SOIL}

\author{
By DR. A. C. THAYSEN
}

Coloniał Microbiological Research Institute, Trinidad

$\mathrm{I}^{\mathrm{N}}$ Wis presidential address in 1949 to the British Association on the subject of world population and world food supplies, Sir John Russell ${ }^{1}$ referred to certain observations made during the year on the isola. tion of antibiotic-producing organisms from tropical soils ${ }^{2}$. Russell suggested that it might eventually become possible to control some soil-borne plant diseases by introducing or encouraging micro-organisms with appropriate antibiotic properties to grow in infected soils.

This stimulating thought, could it but be put into practice, would at once raise the subject of antibiotics from what Conn ${ }^{3}$ described as an interesting, though not an agricultural one, to one of deep agricultural significance and therefore of great interest to soil microbiology. But before such preventive measures could be successfully undertaken, a much better understanding would have to be acquired, not only of the occurrence of antibiotic-producing micro- organisms in soils and in their other natural habitats, but also of the conditions-if they can be shown to exist-under which their activity is favoured.

Without wishing to belittle the efforts made hitherto, it is probably not exaggeration to say that, until quite recently, workers interested in antibiotics concentrated their energies on detecting new types of organisms producing them; on determining the chemical structure of the isolated antibiotics; and on applying the pure or purified substances in combating human and animal infections. Little attention was paid to the natural habitats of micro-organisms with antibiotic properties, or to their functions within these habitats. An exception should be made in the case of Nakhimouskaia's observation ${ }^{4}$ that, as quoted by Waksman ${ }^{5}$, actinomycetes which produce antagonistic substances against other micro-organisms occur naturally more often and in larger numbers in light than in heavy soils, and that the addition of organic matter such as peptone to light soils temporarily reduces the antagonistic activity.

Alexopoulos's attempts ${ }^{6}$ to correlate the reported resistance of certain Honduran soils to Panama disease with the occurrence in such soils of microorganisms producing fungistatic metabolic products can scarcely be regarded as relevant. The same may be said for Meredith's subsequent work ${ }^{7}$ in Jamaica on similar lines. For though certain actinomycetes were isolated in both cases, and the cultures of these organisms were shown to inhibit the growth of the Panama disease fungus, Fusarium oxysporum cubense, in neither case was any correlation brought to light between the numbers and types of actinomycetes found in resistant soils, and the presence or absence of Fusarium oxysporum cubense there. Nor was it indicated why a resistant soil should encourage the growth of the inhibiting strains of actinomycetes, or be a natural habitat for them.

Perhaps these difficulties should not be unduly stressed, for little was known at the time about antibiotics. The actual substance secreted by one of Meredith's actinomycetes was not identified as an antibiotic until $1945^{8}$, and not obtained as a chem. ically pure substance until $1948^{\circ}$.

Even though it be of a restricted nature, some information on the likely function of antibioticproducing micro-organisms in a natural habitat is contained in the observations of Oxford and Singh ${ }^{10}$ on the nutritional requirements of myxobacteria. These workers found that, in artificial cultures, certain myxobacteria secrete an antibiotic which inhibits the growth of, and kills, the cells of Staphylococcus aureus in preparation for their resolution and digestion by the myxobacteria. Since myxobacteria may now be regarded as true soil inhabitants ${ }^{11}$, and since there is little likelihood that the behaviour of these organisms in artificial cultures should be fundamentally different from what it is in their natural habitat, there would here appear to be an isolated case in which a group of soil organisms utilizes the faculty of antibiotic production for its own maintenance, and incidentally also for the suppression of other soil organisms living within the same environment. As such this observation is far more interesting than the recent evidence by Grossbard ${ }^{12}$ that fungi and actinomycetes can produce antibiotics in soils mixed with straw or glucose.

When evidence of the part played by antibiotics in soils has become stronger, the most impressive positive contributions will probably have been derived from studies of closer knitted microbial soil 